Mineral Resources Program

Energy Resources Program

\title{
Geochemical and Mineralogical Properties of Boquillas Shale Geochemical Reference Material ShB00-1
}

The ShBOQ-1 geochemical reference material is relevant to studies of the organic geochemistry and mineralogy of petroleum source rocks containing high concentrations of carbonate minerals and organic sulfur-rich, oil-prone marine organic matter. ShBOQ-1 is geochemically and mineralogically similar to the lower part of the Upper Cretaceous Eagle Ford Shale.

\section{Introduction}

Since 1951, the U.S. Geological Survey (USGS) has developed a number of geochemical reference materials (GRMs) relevant to a wide range of geologic resources. These GRMs provide quality control and experimental materials for USGS researchers as well as for users in industry, academia, and other government agencies. As part of this long tradition of identifying, preparing, and characterizing GRMs, the USGS Mineral Resources Program's Geochemical Reference Materials Project has collaborated with the USGS Energy Resources Program's Petroleum Geochemistry Research Laboratory Project to collect GRMs representative of source rocks from major domestic petroleum systems (Birdwell and others, 2016; Birdwell and Wilson, 2017). These GRMs are meant primarily for use in supporting analytical efforts to characterize the geochemistry and mineralogy of organic-rich mudrocks from petroliferous basins in the United States and around the world.

The GRM data presented here are from the Upper Cretaceous Boquillas Formation (ShBOQ-1) in southwest Texas. The Upper Cretaceous Boquillas Formation (ShBOQ-1) geochemical reference material is relevant to studies of the organic geochemistry and mineralogy of petroleum source rocks containing high concentrations of carbonate minerals and organic sulfur-rich, oil-prone marine organic matter. ShBOQ-1 is geochemically and mineralogically similar to the lower part of the Upper Cretaceous Eagle Ford Shale, which is a major petroleum resource play located in South Texas. The GRM is primarily relevant to marine carbonate source rocks present in a wide range of petroleum systems.

For development of this GRM, a total of approximately 720 kilograms $(\mathrm{kg})$ of carbonate source rock were collected in 2015 at a road cut located approximately 20 miles west of Comstock, Texas, along State Highway $90\left(29^{\circ} 42^{\prime} 10.0^{\prime \prime} \mathrm{N}\right.$, $\left.101^{\circ} 12^{\prime} 40.1^{\prime \prime} \mathrm{W}\right) ; 360 \mathrm{~kg}$ of this material was processed for use in the preparation of ShBOQ-1. The ShBOQ-1 GRM has undergone laboratory testing to quantify (1) major and trace element concentrations, (2) total organic carbon (TOC) content, (3) programmed pyrolysis (for example, Rock Eval) parameters, and (4) X-ray diffraction (XRD) mineralogy.

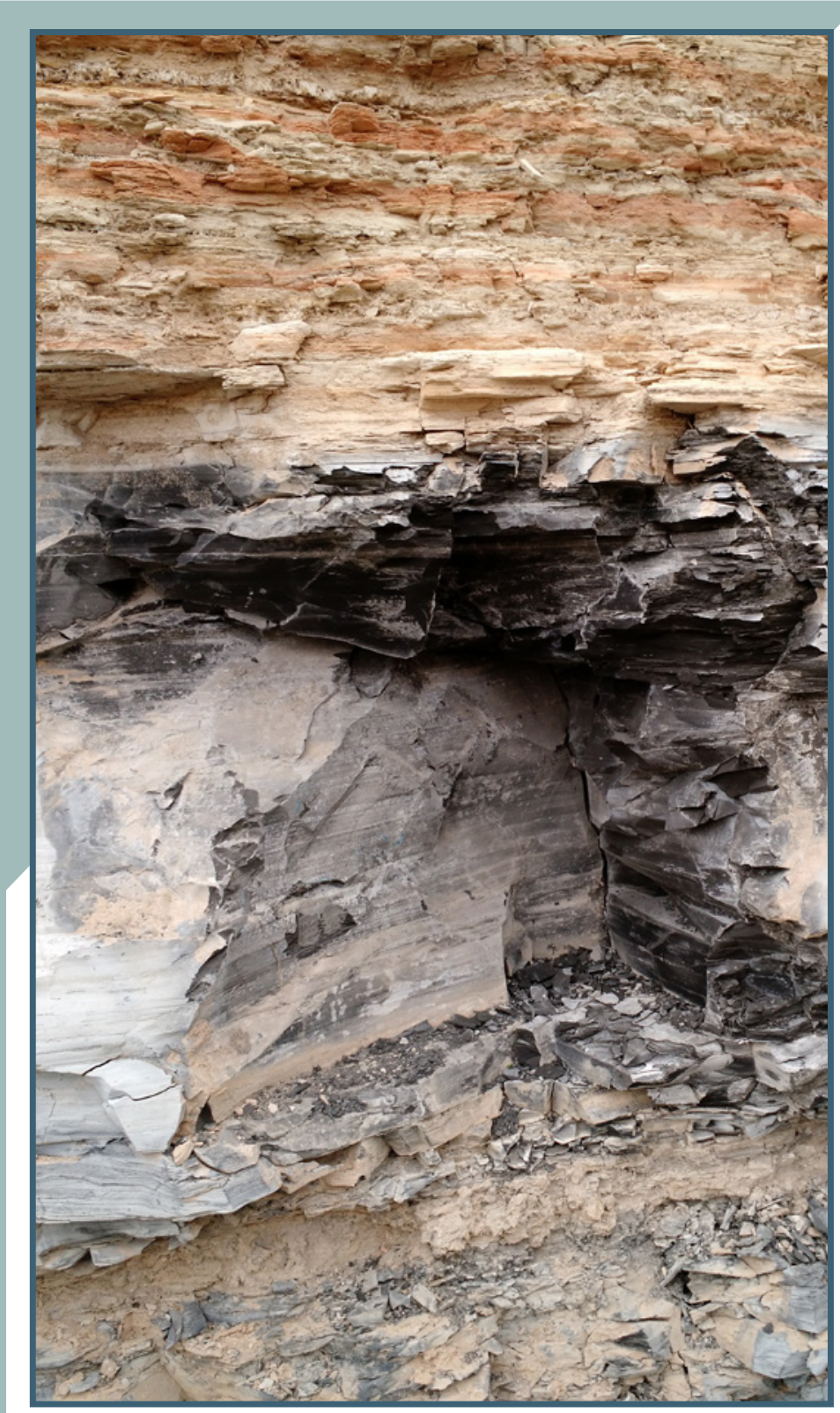

Photograph from a road cut off of US-90 west of Del Rio in Val Verde County, Texas, after collection of ShB0Q-1 material. Photograph by Justin E. Birdwell, U.S. Geological Survey, 2015. 


\section{Testing Study Results}

A number of commercial, academic, and government laboratories participated in interlaboratory testing studies to generate the averages and standard deviations for a variety of source rock properties presented here. Full datasets are available in an accompanying data release (Birdwell and Wilson, 2021). Major and trace element results from 99 laboratories participating in an international testing study using this GRM conducted by the International Association of Geoanalysts (Webb and others, 2017) demonstrate the homogeneity of the ShBOQ-1 GRM. The results from that study indicate that the reported variability in organic geochemical and mineralogical properties are due primarily to analytical variance and not GRM heterogeneity (Birdwell and Wilson, 2019). Two versions of the material were prepared to accommodate laboratory analysis $(<0.074$ millimeters $[\mathrm{mm}])$ and well-head studies $(<4 \mathrm{~mm})$. The data presented here are applicable to both.

Data from a USGS contract laboratory were used to calculate average values and standard deviations for the elemental concentrations presented here based on replicate analyses using a variety of USGS Minerals Resources Program (MRP) analytical methods (U.S. Geological Survey Mineral Resources Program, 2020). Fourteen replicate analyses were made by each method; these results are summarized in table 1 for major elements and other bulk parameters and in table 2 for selected trace elements. These results are consistent with those reported by Webb and others (2017). The full dataset for the elemental results and other properties summarized in this fact sheet can be found in the accompanying USGS data release (Birdwell and Wilson, 2021).

Table 1. Major element concentrations. Major element concentrations were determined using Mineral Resources Program (MRP) Methods 3, 4, 15, 16, 17, and 18 (U.S. Geological Survey Mineral Resources Program, 2020).

[wt. \%, weight percent; LOI, loss on ignition]

\begin{tabular}{lccc}
\hline $\begin{array}{c}\text { Major element oxide and } \\
\text { other parameters }\end{array}$ & $\begin{array}{c}\text { Average } \\
\text { (wt. \%) }\end{array}$ & $\begin{array}{c}\text { Standard } \\
\text { deviation }\end{array}$ & $\begin{array}{c}\text { Number of } \\
\text { analyses }\end{array}$ \\
\hline Silicon dioxide $\left(\mathrm{SiO}_{2}\right)$ & 26.21 & 1.08 & 42 \\
Aluminum oxide $\left(\mathrm{Al}_{2} \mathrm{O}_{3}\right)$ & 4.35 & 0.26 & 56 \\
Iron (III) oxide, total $\left(\mathrm{Fe}_{2} \mathrm{O}_{3} \mathrm{~T}\right)$ & 1.60 & 0.05 & 56 \\
Calcium oxide $(\mathrm{CaO})$ & 33.79 & 0.90 & 56 \\
Magnesium oxide $(\mathrm{MgO})$ & 0.42 & 0.03 & 56 \\
Potassium oxide $\left(\mathrm{K}_{2} \mathrm{O}\right)$ & 0.42 & 0.06 & 56 \\
Sodium oxide $\left(\mathrm{Na}_{2} \mathrm{O}\right)$ & 0.04 & 0.01 & 42 \\
Phosphorus pentoxide $\left(\mathrm{P}_{2} \mathrm{O}_{5}\right)$ & 0.10 & 0.01 & 56 \\
Titanium dioxide $\left(\mathrm{TiO}_{2}\right)$ & 0.18 & 0.01 & 56 \\
Total sulfur $\left(\mathrm{S}_{\text {tot }}\right)^{1}$ & 1.64 & 0.02 & 14 \\
Total carbon $\left(\mathrm{C}_{\text {tot }}\right)^{2}$ & 11.26 & 0.05 & 14 \\
Inorganic carbon $\left(\mathrm{C}_{\text {inorg }}\right)^{3}$ & 6.65 & 0.08 & 14 \\
Organic carbon $\left(\mathrm{C}_{\text {org }}\right)^{4}$ & 4.61 & 0.11 & 14 \\
LOI ${ }^{5}$ & 31.81 & 0.10 & 14 \\
\hline
\end{tabular}

${ }^{1}$ Total sulfur by MRP Method 3.

${ }^{2}$ Total carbon by MRP Method 3 .

${ }^{3}$ Inorganic carbon by MRP Method 4.

${ }^{4}$ Organic carbon by difference $\left(\mathrm{C}_{\text {tot }}-\mathrm{C}_{\text {inorg }}\right)$.

${ }^{5}$ Loss on ignition by MRP Method 15 .
Table 2. Selected trace element concentrations. Trace element concentrations were determined using Minerals Resource Program Methods 6, 15, 16, 17, and 18 (U.S. Geological Survey Mineral Resources Program, 2020).

[ppm, parts per million or milligram/kilogram]

\begin{tabular}{|c|c|c|c|}
\hline Trace element & $\begin{array}{c}\text { Average } \\
\text { (ppm) }\end{array}$ & $\begin{array}{l}\text { Standard } \\
\text { deviation }\end{array}$ & $\begin{array}{c}\text { Number of } \\
\text { analyses }\end{array}$ \\
\hline Arsenic (As) & 14.6 & 0.4 & 28 \\
\hline Barium (Ba) & 73.0 & 4.1 & 42 \\
\hline Beryllium (Be) & 0.7 & 0.1 & 14 \\
\hline Bismuth (Bi) & 0.2 & 0.03 & 28 \\
\hline Cadmium (Cd) & 2.1 & 0.1 & 28 \\
\hline Cerium (Ce) & 23.9 & 0.3 & 28 \\
\hline Cobalt (Co) & 5.9 & 0.2 & 28 \\
\hline Chromium (Cr) & 30.3 & 1.9 & 28 \\
\hline Cesium (Cs) & 3.2 & 0.2 & 14 \\
\hline Copper $(\mathrm{Cu})$ & 37.7 & 1.1 & 28 \\
\hline Dysprosium (Dy) & 1.6 & 0.1 & 14 \\
\hline Erbium (Er) & 0.9 & 0.04 & 14 \\
\hline Europium (Eu) & 0.5 & 0.02 & 14 \\
\hline Gallium (Ga) & 6.1 & 0.6 & 28 \\
\hline Gadolinium (Gd) & 2.0 & 0.04 & 14 \\
\hline Holmium (Ho) & 0.3 & 0.01 & 14 \\
\hline Lanthanum (La) & 13.0 & 0.2 & 28 \\
\hline Lithium (Li) & 26.7 & 2.1 & 28 \\
\hline Lutetium (Lu) & 24.7 & 0.8 & 14 \\
\hline Molybdenum (Mo) & 50.9 & 1.4 & 28 \\
\hline Niobium (Nb) & 5.7 & 0.3 & 28 \\
\hline Neodymium (Nd) & 10.7 & 0.2 & 14 \\
\hline Nickel (Ni) & 80.6 & 2.5 & 28 \\
\hline Lead $(\mathrm{Pb})$ & 6.2 & 0.7 & 28 \\
\hline Praseodymium (Pr) & 3.0 & 0.04 & 14 \\
\hline Rubidium (Rb) & 16.7 & 0.8 & 28 \\
\hline Antimony (Sb) & 3.3 & 0.1 & 28 \\
\hline Scandium (Sc) & 3.3 & 0.2 & 14 \\
\hline Samarium (Sm) & 2.1 & 0.1 & 14 \\
\hline Tin (Sn) & 2.4 & 1.5 & 28 \\
\hline Strontium (Sr) & $1,049.1$ & 51.2 & 42 \\
\hline Terbium (Tb) & 0.3 & 0.01 & 14 \\
\hline Thorium (Th) & 2.8 & 0.1 & 28 \\
\hline Thallium (Tl) & 2.9 & 0.2 & 28 \\
\hline Thulium (Tm) & 0.2 & 0.01 & 14 \\
\hline Uranium (U) & 9.1 & 0.4 & 28 \\
\hline Vanadium (V) & 376.4 & 37.3 & 28 \\
\hline Yttrium (Y) & 9.3 & 0.5 & 28 \\
\hline Ytterbium (Yb) & 0.9 & 0.04 & 14 \\
\hline Zinc (Zn) & 105.2 & 4.2 & 28 \\
\hline Zirconium (Zr) & 52.5 & 1.7 & 28 \\
\hline
\end{tabular}


An interlaboratory testing study was conducted to obtain measurements of TOC content by combustion and infrared analysis and to obtain results from a variety of programmed pyrolysis instruments (Rock Eval 2, Rock Eval 6, Source Rock Analyzer, Hydrocarbon Analyzer with Kinetics) using the same standardized temperature program (Espitalie and others, 1977). Programmed pyrolysis also generates measured values that are used to calculate indices relevant to hydrocarbon-generating potential, thermal maturity, and organic matter type (Peters, 1986). The results of these tests (table 3 ) present averages and standard deviations that were determined after removing outlier datapoints. Outliers were identified for each measured parameter by calculating upper and lower limits using the interquartile range (IQR; acceptance range $=$ average $\pm 1.5 \times \mathrm{IQR})$. After removing outliers, the calculations and outlier-removal steps were repeated until all remaining values fell within the acceptance range. This analysis was also performed on the other datasets presented here, but no outliers were identified.

To optimize drilling and hydraulic fracturing operations, in addition to understanding organic properties, it is important to understand mineralogy in unconventional oil and gas plays. Table 4 presents results from interlaboratory tests to determine XRD mineralogy.

Table 3. Organic geochemical measurements and calculated properties from interlaboratory testing study. Extractable organic matter was obtained by extraction with chloroform using a U.S. Geological Survey standard operating procedure.

[wt. \%, weight percent; S, sommet (peak in French); mg, milligram; g, gram; Tmax, maximum temperature; ${ }^{\circ} \mathrm{C}$, degrees Celsius; TOC, total organic carbon; NA, not applicable]

\begin{tabular}{|c|c|c|c|c|}
\hline Parameter & Average & Standard deviation & $\begin{array}{c}\text { Total number of } \\
\text { analyses }\end{array}$ & $\begin{array}{c}\text { Total after outlier } \\
\text { removal }\end{array}$ \\
\hline \multicolumn{5}{|c|}{ Measured values } \\
\hline Total organic carbon, wt. $\%$ & 5.09 & 0.18 & 156 & 152 \\
\hline Extractable organic matter, wt. \% & 0.89 & 0.03 & 14 & NA \\
\hline S1, mg-hydrocarbon/g-rock ${ }^{1}$ & 1.27 & 0.11 & 114 & 112 \\
\hline S2, mg-hydrocarbon/g-rock ${ }^{1}$ & 31.59 & 2.57 & 114 & 108 \\
\hline S3, mg-carbon dioxide/g-rock ${ }^{1}$ & 0.70 & 0.08 & 114 & 90 \\
\hline $\operatorname{Tmax},{ }^{\circ} \mathrm{C}^{1}$ & 424 & 1.1 & 114 & 92 \\
\hline \multicolumn{5}{|c|}{ Calculated parameters ${ }^{1}$} \\
\hline Hydrogen index, S2/TOC $\times 100$ & 594 & 21 & NA & NA \\
\hline Oxygen index, S3/TOC $\times 100$ & 14 & 2 & NA & NA \\
\hline Oil saturation index, $\mathrm{S} 1 / \mathrm{TOC} \times 100$ & 25 & 2 & NA & NA \\
\hline
\end{tabular}

${ }^{1}$ For details on programmed pyrolysis measured and calculated parameters, see Peters (1986).

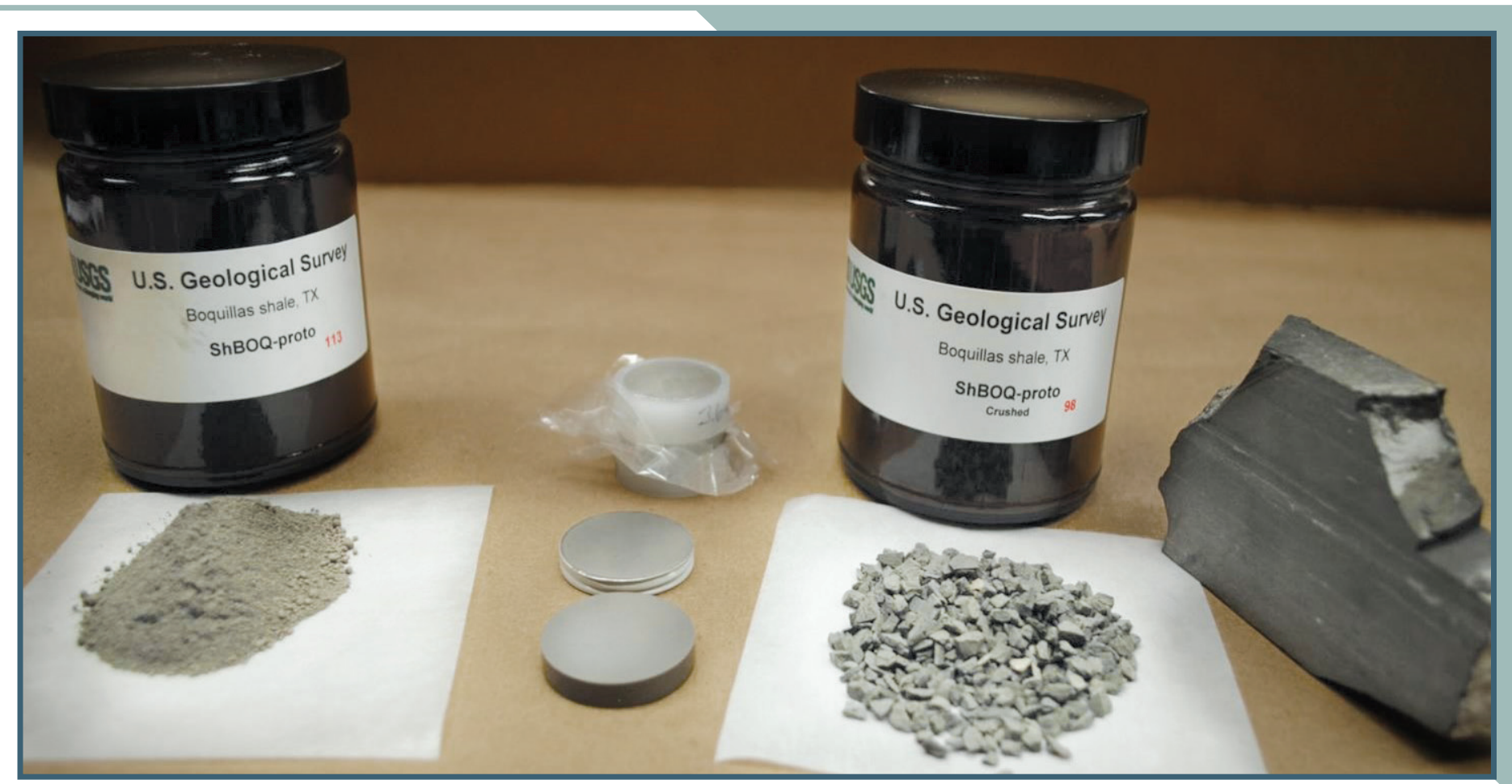

Photograph of preliminary materials ("ShB00-proto") used for initial analyses to confirm Boquillas Shale compositional characteristics prior to interlaboratory testing. From left to right: rock powder, pressed pellets (not available), cutting-size chips, and slab piece.

Photograph by Stephen A. Wilson, U.S. Geologic Survey, 2016. 
Table 4. Mineralogy results from interlaboratory testing study. All values are reported on an organic-free basis and do not account for noncrystalline phases.

[wt. \%, weight percent]

\begin{tabular}{lccc}
\hline Mineral & $\begin{array}{c}\text { Average } \\
\text { (wt. \%) }\end{array}$ & $\begin{array}{c}\text { Standard } \\
\text { deviation }\end{array}$ & $\begin{array}{c}\text { Total number of } \\
\text { detections }\end{array}$ \\
\hline Calcite & 64.4 & 2.9 & 29 \\
Dolomite & 1.1 & 0.8 & 29 \\
Quartz & 22.8 & 2.1 & 29 \\
Kaolinite & 6.9 & 1.9 & 23 \\
Illite/mica & 1.8 & 0.6 & 18 \\
Gypsum & 2.4 & 1.0 & 23 \\
Pyrite & 1.4 & 1.1 & 29 \\
\hline
\end{tabular}

${ }^{1}$ Total number of analyses in which the particular mineral was detected.

\section{Acknowledgments}

The authors would like to thank the participants in the interlaboratory testing studies that generated the data used to generate the values reported in this fact sheet, especially members of the U.S. Geological Survey Petroleum Geochemistry Research Laboratory in Denver, Colorado (Augusta Warden, Mark Dreier, Zack Lowry, Tom Oliver, Rachel Aidala, and Chris Murphy) and the USGS Mineral Resources Program Analytical Chemistry Group in Denver, Colo. (Jaime Azain, Zach Bueghly, and William Benzel). We also thank our colleagues that provided feedback through the review process (Augusta Warden, Paul Lillis, Julie Herrick, Kristen Marra, Ofori Pearson, and Janet Slate).

\section{References Cited}

Birdwell, J.E., and Wilson, S.A., 2017, Development of new shale geochemical reference materials-Results from Boquillas Shale interlaboratory testing and other status updates [abs.], in GSA 2017 Annual Meeting, Seattle, Wash., October 22-25, 2017, Abstracts with programs: Geological Society of America, v. 49, no. 6, paper no. 69-8, 1 p., accessed July 22, 2021, at https://gsa.confex.com/ gsa/2017AM/webprogram/Paper304400.html.
Birdwell, J.E., and Wilson, S.A., 2019, Variability in results from mineralogical and organic geochemical interlaboratory testing of U.S. Geological Survey shale reference materials, in Unconventional Resources Technology Conference, 7th, Denver, Colo., July 22-24, 2019, Proceedings: Unconventional Resources Technology Conference (URTeC), paper no. 457, 19 p., accessed July 22, 2021, at https://archives.datapages.com/ data/urtec/usa/2019/457.htm?q=\%2BtextStrip\%3Abirdwell.

Birdwell, J.E., and Wilson S.A., 2021, Results from geochemical and mineralogical characterization of Boquillas Shale geochemical reference material ShBOQ-1: U.S. Geological Survey data release, https://doi.org/10.5066/P9D6D1KG.

Birdwell, J.E., Wilson, S.A., and Matson, C.C., 2016, Development and applications of new geochemical reference materials to major U.S. unconventional shale plays [abs.], in GSA 2016 Annual Meeting, Denver, Colo., September 25-28, 2016, Abstracts with programs: Geological Society of America, v. 48, no. 7, paper no. 23-4, 1 p., accessed July 22, 2020, at https://gsa.confex.com/gsa/2016AM/webprogram/ Paper283551.html.

Espitalie, J., Madec, M., Tissot, B., Mennig, J.J., and Leplat, P., 1977, Source rock characterization method for petroleum exploration, in the Annual Offshore Technology Conference, 9th, Houston, Tex., May 1-4, 1977, Proceedings: Dallas, Tex., Offshore Technology Conference, paper no. OTC-2935-MS, p. 439-444. [Also available at https://doi.org/10.4043/2935-MS.]

Peters, K.E., 1986, Guidelines for evaluating petroleum source rock using programmed pyrolysis: The American Association of Petroleum Geologists Bulletin, v. 70, no. 3, p. 318-329.

U.S. Geological Survey Mineral Resources Program, 2020, Analytical chemistry, U.S. Geological Survey web page, accessed October 27, 2020, at https://www.usgs.gov/energy-and-minerals/ mineral-resources-program/science/analytical-chemistry.

Webb, P.C., Thompson, M., Potts, P.J., Gowing, C.J.B., and Wilson, S.A., 2017, GeoPT40A-An international proficiency test for analytical geochemistry laboratories-Report on Round 40A (calcareous organic-rich shale, ShTX-1): International Association of Geoanalysts, GeoPT 40A Report, 39 p., accessed July 22, 2021, at http://www.geoanalyst.org/wp-content/ uploads/2018/01/GeoPT40A-Full-report-rev.pdf.
By Justin E. Birdwell and Stephen A. Wilson

For additional information, please contact:
Center Director
USGS Central Energy Resources Science Center
Box 25046, Mail Stop 939
Denver, CO 80225
(303) 236-1647

Or visit:

Central Energy Resources Science Center

website at

https://www.usgs.gov/centers/cersc 